\title{
Measurement of Near-Field Electromagnetic Emissions and Characterization Based on Equivalent Dipole Model in Time-Domain
}

Yuan Zhao, Mohd Hafiz Baharuddin, Christopher Smartt, Xiang Zhao, Liping Yan, Senior Member, IEEE, Changjun Liu, Senior Member, IEEE, David W. P. Thomas, Senior Member, IEEE

\begin{abstract}
In this paper, a method for representing electromagnetic emissions from a device under test (DUT) using an equivalent time dependent dipole array model deduced from the time-domain near-field scanned tangential magnetic fields is proposed. First, a 3D time-domain near-field scanning system is established to measure the tangential magnetic fields emitted from DUTs which are a transmission line above a ground plane and a printed circuit board (PCB) with several microstrips plane and a printed circuit board (PCB) with several microstrips respectively. For time-domain measurements, two magnetic field probes are calibrated over a broad bandwidth for both amplitude and phase to obtain their complex probe factors. Then the measured magnetic fields are utilized to construct an equivalent time dependent dipole array model to represent the electromagnetic sources of the DUT. Parameters of the time dependent equivalent dipoles are directly calculated by fitting to the measured magnetic fields. The effects of different number of dipoles on the accuracy of the reconstructed che PCB are studied. The reconsted magnetic filds from the equivalent dipoles of the electromagnetic fields at other observation levels. The result predicted by the equivalent dipole model are in agreement with the simulated and measured results.

Index Terms-Equivalent dipole model, magnetic fields, printed circuit board, time-domain near-field scanning, transmission line.
\end{abstract}

\section{INTRODUCTION}

ODERN digital systems incorporating some complex V active integrated circuits and passive lumped components work within a variety of environments. Eve increasing clock speeds means that electronic circuits can become unintentional yet efficient radiators, with very complex radiation patterns. Knowledge of the electromagnetic (EM) fields created by these systems and the EM environment in and around these systems is essential for ensuring their efficient and

This work was supported by the COST (European Cooperation in Science and Technology) Action IC1407 "ACCREDIT,", the NSF (Grant No. 61877041) and the NSAF (Grant No. U1530143) of China. (Corresponding author: David W. P. Tho (G.)

Y. Zhao, X. Zhao, L. Yan, and C. Liu are with the College of Electronics and Y. Zhao, X. Zhao, L. Yan, and C. Liu are with the College of Electronics an e zhaoyuanscu@h
cjliu@ieee.org).

M. H. Bhadin is with the Geore Green Institute for Electromagetis Research, University of Nottinghem Nottingham NG7 2RD, UK, and also with the Untre of Universiti Kebangsaan Malaysia, 43600 Bangi, Malaysia (e-mai hafizb@ukm.edu.my).

C. Smartt and D. W. P. Thomas are with the George Green Institute for ElecC. Smetics Research, University of Nottinghm, Nottingham NG7 2RD, U.K. (e-mil: chris smarttenttingham ac.uk; dave thoma@entinghmac.uk). reliable operation which is important in the research field of electromagnetic compatibility (EMC) and electromagnetic interference (EMI). A widely used technique for characterizing EM properties is near-field scanning. This technique is first adopted for antenna measurements [1]. Due to its high measurement accuracy and reliability, the near-field scanning technique has also been developed for emission tests in EMC research [2]-[9]. Adopting near-field scanning, the electric or magnetic fields in the near-field region can be obtained [2]-[4] Usually the transverse fields are measured, as the source can be completely defined by the transverse electric or magnetic fields [2]-[4]. The measured near-fields data are mainly used in three ways. First, the intensities and distribution of the emissions from a device under test (DUT) can be intuitively observed which will reveal the emission property, both radiated and evanescent, of the DUT and hence its potential for interference. Second, the data can be used to reconstruct an equivalent source model of the DUT, which can be incorporated into a system-level simulation, thus deconstructing the radiated and evanescent field components. Third, the data can be used to derive the fields at other observation planes above the measurement plane based on propagation theory [10] or the properties of the equivalent sources [2]-[4], [11]. This will reduce the repeating of measurements to save time [2]-[4]. Conventionally, two kinds of dipole models are usually introduced to depict the emission properties of DUTs. One is a single electric and/or magnetic dipole at the same spot which is suitable for representing an integrated circuit (IC) [12]. The other one is to use a spatially distributed array of electric and/or magnetic dipoles [2]-[9]. Although the proposed equivalent dipoles do not have physical equivalence, as the non-confirmed hypothesis discussed in [18] that magnetic dipole model would perform better than the electric dipole model for a microstrip loop circuit, an accurate reconstruction of the equivalent dipole model can help engineers to propose effective methods to solve the EMC and EMI problems [2].

On the one hand, the near-field scanning technique has been extensively explored in the frequency domain [1]-[9]. On the other hand, measurements and analyses of the near-fields in the time-domain are becoming increasingly important for characterizing the electromagnetic emission properties of the DUT. This is because it is recognized that the interference in many digital systems and communications links are better quantified in the time-domain [13] and also time-domain analysis can be more efficient [14]. For this reason, several
Commented [ZY1]: Respond to Comment 1 from Reviewer 3. I' $\mathrm{m}$ not sure whether this is a proper response or not. 
researchers have proposed time-domain near-field scanning techniques [15]-[20]. In [15], the inverse problem for estimating the equivalent sources is solved using a genetic algorithm and it is not demonstrated that the method can extrapolate them to different heights from the DUT. In [16], experimental verification of the approach given in [17] is provided using frequency domain measurements from a vector network analyzer (VNA). [18] provides a theoretical analysis of a technique estimating an equivalent, time-domain, dipole array that neglects the retarded time between the source dipole which is not appropriate for the very near field. In [19], the mutual coupling between the probe and current bearing tracks is used to measure the currents directly, but this requires detailed knowledge of the track geometry to estimate. In [20], the far-field pattern is estimated using a near to far field transformation.

The proposed approach in this paper is based on equivalent time dependent electric dipole array model which is inherently broadband and can provide information on the time dependent properties of the magnetic fields from the DUT. The methodology presented here directly calculates the inverse problem and was first presented in [17]. Here a more complete description of the methodology and full time-domain experimental verification is presented using a high-speed digital oscilloscope. The initial closest scan plane is used to characterize the DUT and predictions at planes at greater heights from the DUT are verified against measurements.

This paper is organized as follows. Section II discusses the principle of equivalent dipole model theory. Section III introduces the time-domain near-field scanning system and probe calibration. The full-wave simulated, experimental, and predicted results of the emission magnetic fields from a transmission line (TL) above a ground plane and a printed circuit board (PCB) with several microstrips are presented respectively in this section. The conclusions are given in Section IV.

\section{The Theory of EQuivalent Dipole Model}

In this section, a complete description of the equivalent dipole model theory is presented. The basic idea of this approach is to replace the real sources of the DUT with an array of equivalent infinitesimal electric dipoles, consequently, they should reproduce the same fields as the real DUT for regions of space above the scanning plane. For generality, the equivalen dipole model is built without a priori knowledge on the DUT structural details other than its overall geometrical dimensions. The DUT is segmented and represented by an array of arbitrarily orientated dipoles on a planar surface within the area of the DUT. Each elemental point dipole is decomposed into three Cartesian components $D_{x}, D_{y}$, and $D_{z}$. The momen (magnitude and phase) and orientation of the electric dipoles are determined by fitting the measured tangential magnetic fields distribution in a near-field scanning plane from a solution of the inverse problem.

When a DUT is backed by a ground plane, like a PCB, it causes a difficulty in the deriving of the far-field radiation in the space below the ground plane where the near-field is normally too weak to be measured due to the finite dynamic range of the measurement system. To deal with these problems, the finite ground plane is directly included in the equivalent dipole model to account for its diffraction effects. Equivalent dipoles are placed on a surface which is a general distance $h$ above the ground plane, $h$ being the thickness of the PCB or expected height of sources above the ground plane and over an area that is $6 h-10 h$ smaller than the PCB dimensions [3], [4]. Image theory is used in the equivalent dipoles identification which only applies to infinite ground planes. For a finite ground plane, the total field has a diffraction term besides the direct and image terms. But tangential magnetic fields in the reactive near-field above the DUT are a good approximation to the contribution of direct and image radiation, with only a negligible proportion of diffraction [3], [4], [11]. If near-field scans are performed in this region, the ground plane can be assumed to be of infinite size so as to apply image theory in the equivalent dipoles identification procedure.

In Cartesian coordinates, for a DUT in the $x y$ plane, the tangential magnetic fields will be $H_{x}(t)$ and $H_{y}(t)$. The magnetic field at $(x, y, z)$ produced by infinite small dipoles $D_{x}$ $D_{y}$, and $D_{z}$ at $\left(x_{0}, y_{0}, z_{0}\right)$ can be expressed as [17]

$$
\begin{aligned}
H_{x}(t)= & \frac{z-z_{0}}{4 \pi}\left[\frac{1}{r^{3}} D_{y}(t-r / c)+\frac{1}{c r^{2}} \frac{\partial D_{y}(t-r / c)}{\partial(t-r / c)}\right] \\
& -\frac{y-y_{0}}{4 \pi}\left[\frac{1}{r^{3}} D_{z}(t-r / c)+\frac{1}{c r^{2}} \frac{\partial D_{z}(t-r / c)}{\partial(t-r / c)}\right] \\
H_{y}(t)= & \frac{x-x_{0}}{4 \pi}\left[\frac{1}{r^{3}} D_{z}(t-r / c)+\frac{1}{c r^{2}} \frac{\partial D_{z}(t-r / c)}{\partial(t-r / c)}\right] \\
& -\frac{z-z_{0}}{4 \pi}\left[\frac{1}{r^{3}} D_{x}(t-r / c)+\frac{1}{c r^{2}} \frac{\partial D_{x}(t-r / c)}{\partial(t-r / c)}\right]
\end{aligned}
$$

where $r=\sqrt{\left(x-x_{0}\right)^{2}+\left(y-y_{0}\right)^{2}+\left(z-z_{0}\right)^{2}}$ is the distance between magnetic field point and dipole point. $c$ is the speed of light in free space. The term $(t-r / c)$ is the retarded time between the dipole and the field observation point. For large scan heights $z$, all dipole retarded times are approximately $(t-z / c)$. However, for measurement of an extended equivalent dipole array in the near-field, the possible variation in retarded time may be significant and must be included in the analysis. The tangential magnetic fields at each measurement point can be represented by the total contribution of all the equivalent dipoles and each dipole $D_{\alpha}$ having three Cartesian components. Suppose there are $m$ measurement sampling points and $n$ equivalent electric dipoles, the measured tangential magnetic fields in the scanning planar array of discrete points are therefore related to the array of equivalent electric dipoles. Each dipole has a different separation distance $r$ from each magnetic field observation point. In order to calculate $D_{x}, D_{y}$, and $D_{z},(1)$ and (2) can be converted to a matrix form (3) 
$\left[\begin{array}{l}H_{x}(t) \\ H_{y}(t)\end{array}\right]=\sum_{\alpha}\left([g]\left[\begin{array}{c}D_{\alpha}\left(t-\frac{r}{c}\right) \\ \frac{\partial D_{\alpha}\left(t-\frac{r}{c}\right)}{\partial\left(t-\frac{r}{c}\right)}\end{array}\right]+\left[g^{\prime}\right]\left[\begin{array}{c}D_{\alpha}\left(t-\frac{r}{c}\right) \\ \left.\frac{\partial D_{\alpha}\left(t-\frac{r}{c}\right)}{\partial\left(t-\frac{r}{c}\right)}\right]\end{array}\right)\right.$

where $D_{\alpha}=\left[D_{x} D_{y} D_{z}\right]^{T}$. The matrix $[G]=[g]+\left[g^{\prime}\right]$, can be written in the form $\left[\begin{array}{ll}g_{s} & g_{c}\end{array}\right]$, where $\left[g_{s}\right]$ and $\left[g_{c}\right]$ is expressed as (4) and (5), $[\mathrm{M}]^{T}$ is the transpose of matrix $[\mathrm{M}]$ and $\alpha$ is the dipole index

$\left[g_{s}\right]$

$=\left[\begin{array}{ccc}0 & \frac{z-z_{0}}{4 \pi r^{3}}-\frac{z+z_{0}}{4 \pi r^{\prime 3}} & -\frac{y-y_{0}}{4 \pi r^{3}}-\frac{y-y_{0}}{4 \pi r^{\prime 3}} \\ -\frac{z-z_{0}}{4 \pi r^{3}}+\frac{z+z_{0}}{4 \pi r^{\prime 3}} & 0 & \frac{x-x_{0}}{4 \pi r^{3}}+\frac{x-x_{0}}{4 \pi r^{\prime 3}}\end{array}\right]$

$\left[g_{c}\right]$

$=\left[\begin{array}{ccc}0 & \frac{z-z_{0}}{4 \pi c r^{2}}-\frac{z+z_{0}}{4 \pi c r^{\prime 2}} & -\frac{y-y_{0}}{4 \pi c r^{2}}-\frac{y-y_{0}}{4 \pi c r^{\prime 2}} \\ -\frac{z-z_{0}}{4 \pi c r^{2}}+\frac{z+z_{0}}{4 \pi c r^{\prime 2}} & 0 & \frac{x-x_{0}}{4 \pi c r^{2}}+\frac{x-x_{0}}{4 \pi c r^{\prime 2}}\end{array}\right]$

where $r^{\prime}=\sqrt{\left(x-x_{0}\right)^{2}+\left(y-y_{0}\right)^{2}+\left(z+z_{0}\right)^{2}}$ is the distance between magnetic field point and image dipole point. In this work, a regular time sampling period, $\Delta t$, is used and thus the dipole estimate can be reduced to a readily solvable form. It is assumed that the recorded data can only be approximated to within one sample period such that the retarded time $r / c$ is also approximated to the nearest integer multiple of the sample period

$$
\frac{r}{c}=k \Delta t, k=0,1,2, \ldots, K
$$

The time derivative is approximated by (7)

$$
\frac{\partial D_{\alpha}(t)}{\partial t}=\frac{D_{\alpha}(t)-D_{\alpha}(t-\Delta t)}{\Delta t}
$$

Applying (4), (5), (6), and (7) to (3) gives (8)

$$
\begin{aligned}
{\left[\begin{array}{l}
H_{x}(t) \\
H_{y}(t)
\end{array}\right]=\sum_{\alpha}([} & \left.g_{s}+\frac{g_{c}}{\Delta t}\right]\left[D_{\alpha}(t-k \Delta t)\right] \\
& \left.-\left[\frac{g_{c}}{\Delta t}\right]\left[D_{\alpha}(t-k \Delta t-\Delta t)\right]\right) .
\end{aligned}
$$

At each measurement point, the tangential magnetic field can be represented by the total contribution of all the equivalent dipoles.

$$
\begin{aligned}
{\left[\begin{array}{l}
H_{x}(t) \\
H_{y}(t)
\end{array}\right]=} & \sum_{\alpha}\left\{\left[g_{s}+\frac{g_{c}}{\Delta t}\right]_{1}\left[D_{\alpha}(t)\right]\right. \\
& +\left(\left[g_{s}+\frac{g_{c}}{\Delta t}\right]_{2}-\left[\frac{g_{c}}{\Delta t}\right]_{1}\right)\left[D_{\alpha}(t-\Delta t)\right] \\
& +\left(\left[g_{s}+\frac{g_{c}}{\Delta t}\right]_{3}-\left[\frac{g_{c}}{\Delta t}\right]_{2}\right)\left[D_{\alpha}(t-2 \Delta t)\right] \\
& +\cdots \\
& \left.+\left(-\left[\frac{g_{c}}{\Delta t}\right]_{K}\right)\left[D_{\alpha}(t-K \Delta t)\right]\right\} .
\end{aligned}
$$

The number of terms $K$ in (9) depends on the maximum value of the retarded time which depends on the size of the DUT, the size of equivalent dipole array, and the scanning height (i.e. $\left.K=\left(r_{\max }-r_{\min }\right) / c \Delta t\right)$. At each time step, the equivalent dipole $D_{\alpha}$ can be deduced from the solution of (10)

$$
\begin{aligned}
{\left[D_{\alpha}(t)\right] } & =\left[g_{s}+\frac{g_{c}}{\Delta t}\right]_{1}^{-1}\left(\left[\begin{array}{l}
H_{x}(t) \\
H_{y}(t)
\end{array}\right]\right. \\
& +\sum_{\alpha}\left\{\left(\left[\frac{g_{c}}{\Delta t}\right]_{1}-\left[g_{s}+\frac{g_{c}}{\Delta t}\right]_{2}\right)\left[D_{\alpha}(t-\Delta t)\right]\right\} \\
& +\sum_{\alpha}\left\{\left(\left[\frac{g_{c}}{\Delta t}\right]_{2}-\left[g_{s}+\frac{g_{c}}{\Delta t}\right]_{3}\right)\left[D_{\alpha}(t-2 \Delta t)\right]\right\} \\
& +\cdots \\
& \left.+\sum_{\alpha}\left\{\left[\frac{g_{c}}{\Delta t}\right]_{K}\left[D_{\alpha}(t-K \Delta t)\right]\right\}\right) .
\end{aligned}
$$

The measured tangential magnetic fields $H_{x}(t)$ and $H_{y}(t)$ with the estimated equivalent dipoles from the previous time step $D_{\alpha}(t-\Delta t)$ are used to calculate the dipoles $D_{\alpha}(t)$. The solution therefore requires that the initial fields are zero. To obtain a unique solution for $D_{\alpha}(t)$, the total number of equivalent dipoles must not exceed the total number of measured magnetic field points. With acenrate near field measurement, the equivalent dipoles can fully characterize the emission properties of the DUT for the half space above the scan plane, provided the plane is large enough to include all the significant fields [3], [4]. This can include predicting fields on the planes on the side of the DUT but only for the region above the original sean plane.

The main steps of the proposed method are described by flowchart in Fig. 1. Initially, the number and locations of the elementary equivalent electric dipoles are predefined. Then, the dipole parameters are determined by (10) with the measured magnetic fields data. Then, the prediction accuracy can be assessed by the relative-error function in (11) to determine the final suitable equivalent dipole array.

The relative-error function $\varepsilon$ between the magnetic fields generated by the equivalent dipole model $H_{\xi d}(\mathrm{t})$ and the measured magnetic fields $H_{\xi}(\mathrm{t})$ are calculated for each instant of time with the following formula (11)

$$
\varepsilon=\frac{\sqrt{\sum_{n_{x}, n_{y}=1,1}^{N_{x}, N_{y}}\left|H_{\xi d}\left(n_{x}, n_{y}, t\right)-H_{\xi}\left(n_{x}, n_{y}, t\right)\right|^{2}}}{\sqrt{\sum_{n_{x}, n_{y}=1,1}^{N_{x}, N_{y}}\left|H_{\xi}\left(n_{x}, n_{y}, t\right)\right|^{2}}}
$$

where $\xi=(x, y)$. 


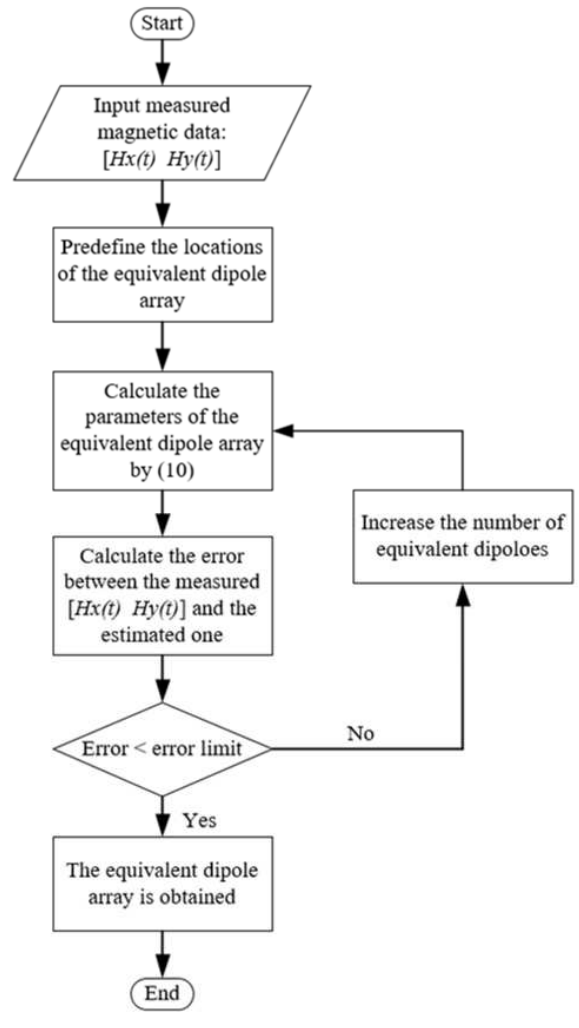

Fig. 1. Flowchart of the proposed method.

III. EXPERIMENTAL AND PREDICTED RESULTS

In this section, the time-domain near-field scanning system is established first. And the full-wave simulated, experimental, and predicted results of the emission magnetic fields from a TL above a ground plane and a PCB with several microstrips are presented respectively to demonstrate the validity of the measurement system and the proposed method.

\section{A. Probe Calibration}

A typical near-field scanning system consists of a probe, a probe positioning and moving subsystem, and a data receiving and processing unit as shown in Fig. 2 [21]
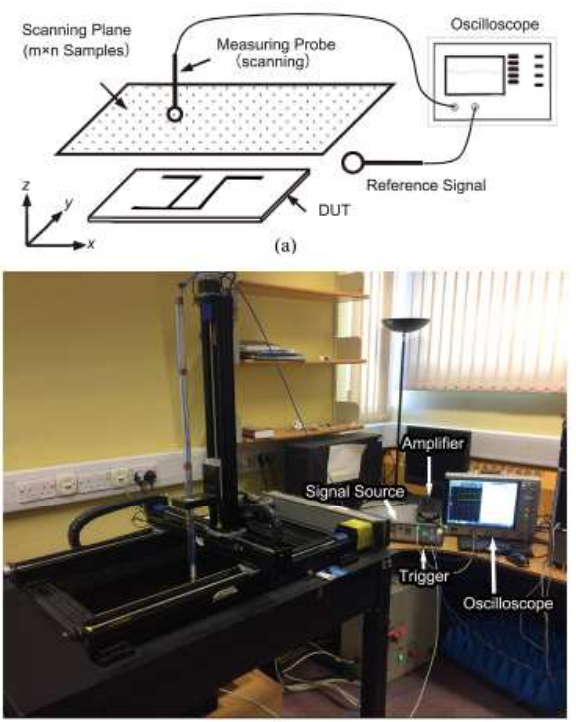

(b)

Fig. 2. The time-domain near-field scanning system. (a) Structural diagram. (b) In the University of Nottingham.

Typically, in time-domain measurement, a single scanning probe is used with a reference signal, for example the trigger signal output from the function generator which used on the oscilloscope to synchronize the sampling of the magnetic fields from the DUT. During the actual measurement procedure, a 20 $\mathrm{dB}$ amplifier and a $-3 \mathrm{~dB}$ attenuator added to improve the impedance matching of the system.

Another important part for time-domain near-field scanning is the calibration of the probe. As we need to determine the time response of the field from the measured time-domain probe voltage we require a complex frequency domain calibration so as to include phase information. A DUT consists of a double loop structure is designed to provide a known fields value needed for the calibration process. The first loop is an identical single wire which emerges from the splitter designed on the DUT. The wire will go up to a height of $40 \mathrm{~mm}$ and will travel $40 \mathrm{~mm}$ and finally will go down and connect to the ground plane. The second loop will have the same dimension as the first loop but will start from the opposite end of the splitter. This will create two loops that are separated by a distance of 40 $\mathrm{mm}$. The source connected to the splitter is coming from Port 1 of a VNA while port 2 is connected to the probe under test. The magnetic field probe will be positioned directly in between the double loop structure as shown in Fig. 3 and $S_{21}$ reading of the VNA will be recorded for a frequency range of $30 \mathrm{MHz}$ to $3 \mathrm{GHz}$. The structure was designed to produce a predictable field with minimum spatial variation over the probe loop area to minimize position error uncertainty. 


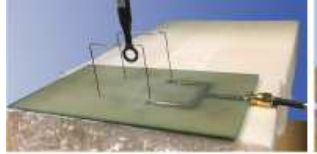

(a)

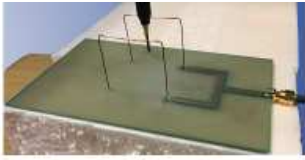

(b)

Fig. 3. A DUT cos probe RF-R50-1. (b) With probe RF-R0,3-3.

In order to obtain the complex probe factor $(\mathrm{PF})$, a model of the DUT was established from full-wave simulation. The accurate magnetic field information $H(f)$ obtained from full-wave simulation are divided by the measured induced voltage on the probe $U(f)$ to obtain a frequency dependent $P F(f)$ as calculated in (12)

$$
P F(f)=20 \log \frac{H(f)}{U(f)} .
$$

The complex $P F(f)$ is a unique function for each probe and measurement set-up which includes the insertion loss of the system. The PFs obtained by the structure are given in Fig. 4.
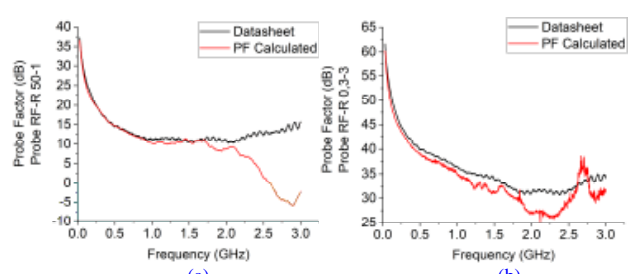

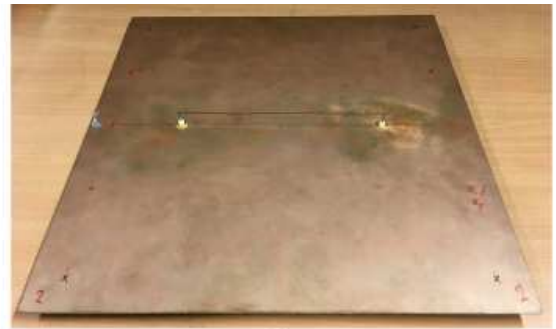

(a)

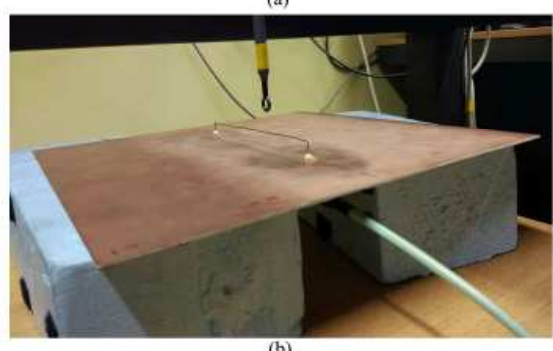

(b)

Fig. 5. A single TL above a ground plane. (a) Top view of the DUT. (b) The DUT placed in near-field scanning system.

The TL is excited by a differential Gaussian pulse with a pulse repetition rate of $20 \mathrm{MHz}$ which is provided by a function generator. The shape of the excitation signal is shown in Fig. 6 .
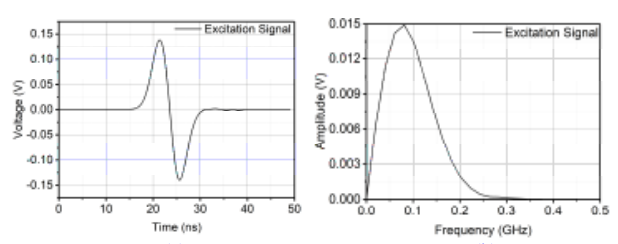

Fig. 6. The excitation signal. (a) Time response of the excitation signal. (b) Fig. 6. The excitation signal. (a) Time resp
Frequency response of the excitation signal.

A Langer EMV-Technik RF-R50-1 magnetic field probe (10 $\mathrm{mm}$ in diameter) [22] is adopted to measure the tangential magnetic fields from the TL in $x$-direction and $y$-direction respectively. The probe initially locates at the start point of the scanning area which is at the red spot and stops at the blue spot as shown in Fig. 7. The fields out of the scan area drop to a low enough level that they can be assumed to be zero. 


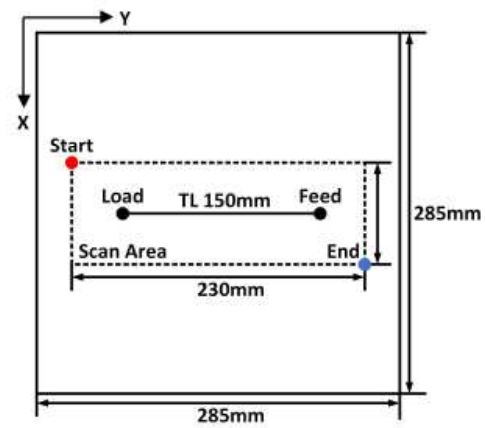

Fig. 7. The scan area of the TL above the ground plane.

The scanning of the tangential magnetic fields in $x$ - and $y$-direction are completed for two scan heights of $5 \mathrm{~mm}$ and $10 \mathrm{~mm}$ above the TL respectively with the sample grid spacing $5 \mathrm{~mm}$. Each plane is measured with $17 \times 47$ sampling points The induced voltage output of the probe is recorded at $4 \mathrm{GSa} /$ second using a digital oscilloscope. The fast Fourie transform (FFT) of the voltage output of the probe in time-domain gives the corresponding frequency domain signal which multiply the $P F(f)$ to obtain the magnetic field information. The inverse fast Fourier transform (IFFT) i performed to reconstruct the time-domain magnetic fields. The tangential magnetic fields are estimated by the equivalent dipole model with a $3 \times 21$ dipole array over the ground plane. In the $x$-direction, the 3 dipoles are equidistantly placed from 0.03 $\mathrm{m}$ to $0.05 \mathrm{~m}$. In the $y$-direction, the 21 dipoles are equidistantly placed from $0.03 \mathrm{~m}$ to $0.21 \mathrm{~m}$. In all of the following results, the dipole moments are given by the fields measured at the lower height.

In order to validate the accuracy of the experimental procedure and the proposed method, an observation point, which is at the middle of TL ( $x=142.5 \mathrm{~mm}, \mathrm{y}=142.5 \mathrm{~mm})$ and $5 \mathrm{~mm}$ above the TL, is selected to compare the measured and estimated tangential magnetic fields (magnetic fields in $x$-direction denoted as $H_{x}(t)$, magnetic fields in $y$-direction denoted as $H_{y}(t)$, at the center of the probe) over the time range in Fig. 8 and good agreements are obtained. The deviation in $H_{y}(t)$ fields is possibly due to positioning error. The results also demonstrate the prediction of propagation away from the lower reference plane.

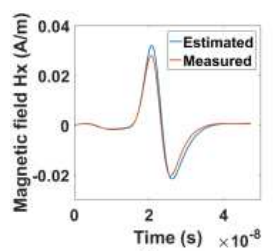

(a)
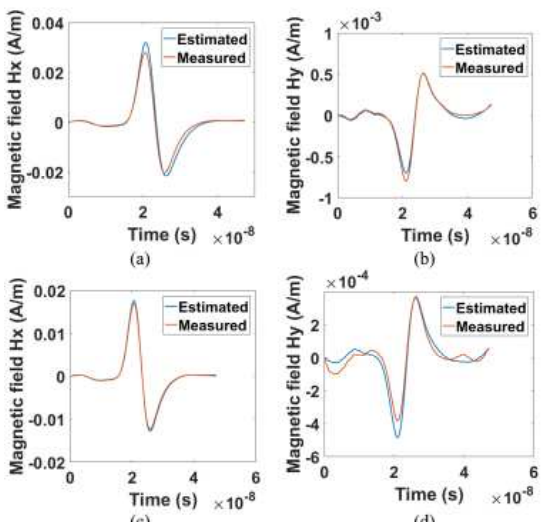

(b)

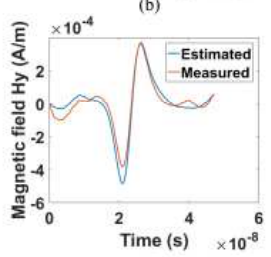

Fig. 8. The measured and estimated tangential magnetic fields of observation point at two scanning heights. (a) $H_{x}(t)$ above TL $5 \mathrm{~mm}$. (b) $H_{y}(t)$ above TL $5 \mathrm{~mm}$. (c) $H_{x}(t)$ above TL $10 \mathrm{~mm}$. (d) $H_{y}(t)$ above TL $10 \mathrm{~mm}$.

The tangential magnetic fields $H_{x}(t)$ and $H_{y}(t)$ in $x y$ plane at the two scanning heights are shown in Fig. 9 to Fig. 10. In Fig. 9, the tangential magnetic fields estimated by the equivalent dipole model with a $3 \times 21$ dipole array over the ground plane are shown in the subfigures (e) and (f). Note that the dipole array is deduced from the lower height of $5 \mathrm{~mm}$. The measured tangential magnetic fields are shown in the subfigures (c) and (d). The full-wave simulated tangential magnetic fields are also given as references in the subfigures (a) and (b) to validate the measured and estimated results. From the comparison, the magnetic fields and their propagation can be well predicted by the equivalent dipole model. Furthermore, these equivalent dipoles can then be used to predict the tangential magnetic fields at other heights, which are greater than the reference plane of $5 \mathrm{~mm}$ height. Two different sampling time points, $\mathrm{t} 1=21.3 \mathrm{~ns}$ and $\mathrm{t} 2=25.6 \mathrm{~ns}$, are selected to present the results.
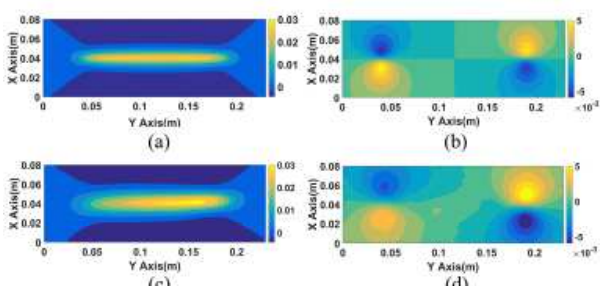
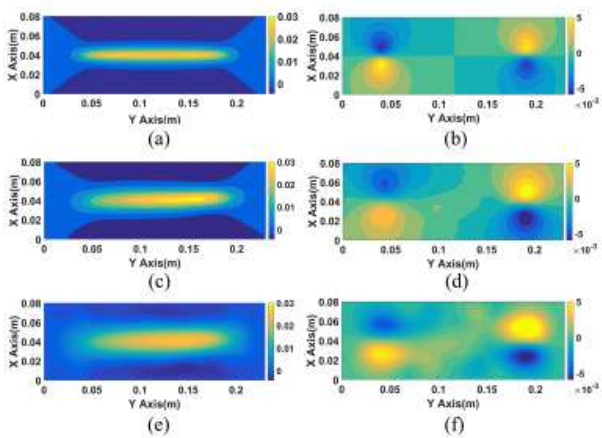

Fig. 9. Tangential magnetic fields $(\mathrm{A} / \mathrm{m})$ in the $x y$ plane at $\mathrm{t}=21.3 \mathrm{~ns}$ at scanning height $5 \mathrm{~mm}$ above the TL. (a) Full-wave simulated $H_{x}(t)$. (b) Full-wave sight $5 \mathrm{~mm}$ above the TL. (a) Full-wave simulated $H_{x}(t)$. (b) Full-wave
simulated $H_{y}(t)$. (c) Measured $H_{x}(t)$. (d) Measured $H_{y}(t)$. (e) Estimated $H_{x}(t)$. (f) Estimated $H_{y}(t)$. 

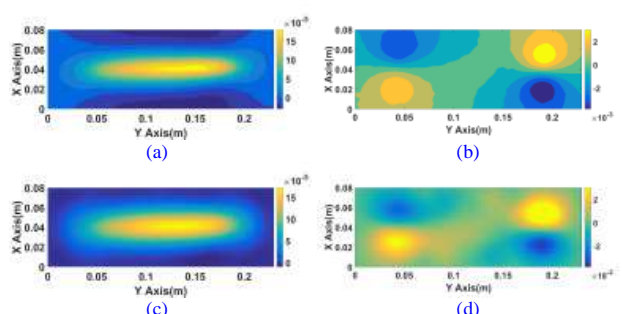
(d)

Fig. 10. Tangential magnetic fields $(\mathrm{A} / \mathrm{m})$ in the $x y$ plane at $\mathrm{t}=21.3 \mathrm{~ns}$ at scanning height $10 \mathrm{~mm}$ above the TL. (a) Measured $H_{x}(t)$. (b) Measured $H_{y}(t)$ (c) Estimated $H_{x}(t)$. (d) Estimated $H_{y}(t)$. Note (c) and (d) use the dipoles deduced at $5 \mathrm{~mm}$ above the TL.

Fig. 11 and Fig. 12 are the comparison of the magnetic fields at sampling time $\mathrm{t} 2=25.6 \mathrm{~ns}$ at two different scanning heights.

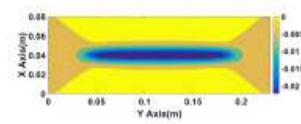

(a)

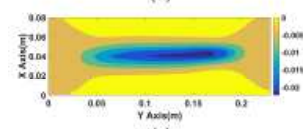

(c)

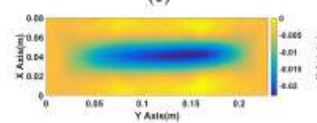

(e)

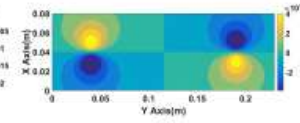

(b)

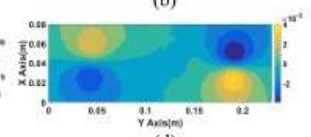

(d)

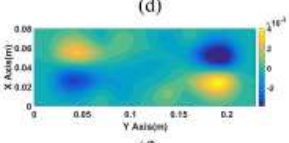

(f)
Fig. 11. Tangential magnetic fields $(\mathrm{A} / \mathrm{m})$ in the $x y$ plane at $\mathrm{t}=25.6 \mathrm{~ns}$ at scanning height $5 \mathrm{~mm}$ above the TL. (a) Full-wave simulated $H_{x}(t)$. (b) Full-wave simulated $H_{y}(t)$. (c) Measured $H_{x}(t)$. (d) Measured $H_{y}(t)$. (e) Estimated $H_{x}(t)$. (f) Estimated $H_{y}(t)$
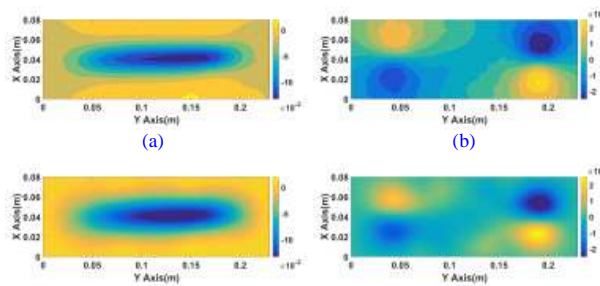

$(\mathrm{c})$
Fig. 12. Tangential magnetic fields $(\mathrm{A} / \mathrm{m})$ in the $x y$ plane at $\mathrm{t}=25.6 \mathrm{~ns}$ at

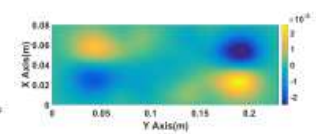
scanning height above TL $10 \mathrm{~mm}$. (a) Measured $H_{x}(t)$. (b) Measured $H_{y}(t)$. (c) Estimated $H_{x}(t)$. (d) Estimated $H_{y}(t)$. Note (c) and (d) use the dipoles deduced at $5 \mathrm{~mm}$ above the $\mathrm{TL}$.

Table I gives the relative-errors between the reconstructed fields and measured fields as given by (11).
TABLE I

THE ReLative-ERrors BetweEn THE MEASURED MAGNetic FIELDS AND THE RECONSTRUCTED ONESFROMTHETRANSMISSION LINE ABOVETTHE

\begin{tabular}{llll}
\multicolumn{4}{c}{ GROUND PLANE } \\
\hline & & $H_{x}(t)$ & $H_{y}(t)$ \\
\hline \multirow{2}{*}{ Scanning Height 5 mm } & $\mathrm{t}=21.3 \mathrm{~ns}$ & $7.94 \%$ & $6.90 \%$ \\
& $\mathrm{t}=25.6 \mathrm{~ns}$ & $6.77 \%$ & $9.32 \%$ \\
Scanning Height 10 mm & $\mathrm{t}=21.3 \mathrm{~ns}$ & $6.73 \%$ & $8.65 \%$ \\
& $\mathrm{t}=25.6 \mathrm{~ns}$ & $7.73 \%$ & $7.94 \%$ \\
\hline \hline
\end{tabular}

From Fig. 9 to Fig. 12 and Table I, good agreements among simulated, measured, and estimated results of magnetic fields from the TL above a ground plane validate the accuracy of the near-field scanning procedure and the effectiveness of the equivalent dipole model to replace the source. The results also demonstrate the effectiveness of predicting the propagation away from the reference plane where the source dipoles are deduced ( $5 \mathrm{~mm}$ above the TL) as from Table I the $10 \mathrm{~mm}$ height has similar errors.

\section{A PCB with Several Microstrips}

In this part, a PCB with several microstrips as shown in Fig. 13 is measured in time-domain with the near-field scanning system and the equivalent dipole model is adopted to reconstruct the sources on the $\mathrm{PCB}$ and estimate the magnetic fields from it.
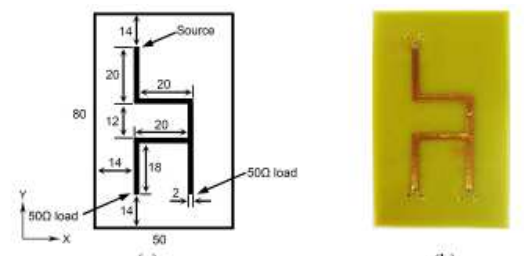

(a)

Fig. 13. Top view of the PCB with several microstrips. (a) Schematic, unit: $\mathrm{mm}$ (b) Photograph

The dimensions of the PCB and the microstrips on its surface are shown in Fig. 13(a): an $80 \mathrm{~mm} \times 50 \mathrm{~mm} \times 1.5 \mathrm{~mm}$ board with FR4 substrate (its relative dielectric constant is 4.35 ), is located in the $x y$ plane. Several $2 \mathrm{~mm}$ wide microstrips are located on one side of the board and a ground plane on the other. At source port, the microstrip is powered by an external voltage source the same as shown in Fig. 6, and the other two load ports are terminated by two $50 \Omega$ loads respectively. The photograph of the PCB is shown in Fig. 13(b).

A Langer EMV-Technik RF-R0,3-3 magnetic field probe (2 $\mathrm{mm}$ in diameter) [23] is adopted to measure the magnetic fields from the PCB. For the specific physical feature of this probe, it can be placed very close to the surface of the PCB board to detect the details of the magnetic fields from the microstrips. It is also calibrated as discussed in Part A. The probe initially locates at the corner of the scanning area which is same as the dimensions of the PCB surface shown in Fig. 14. 


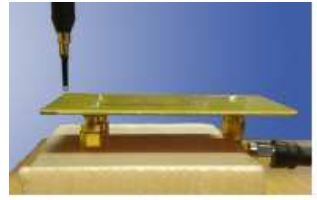

(a)

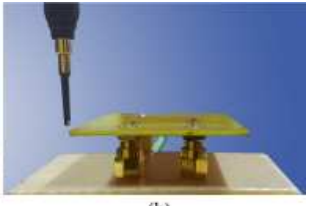

(b)

Fig. 14. The PCB with several microstrips under near-field scanning. (a) The view in $x$-direction. (b) The view in $y$-direction.

During the scanning process, the tangential magnetic fields from the PCB are detected for two scanning heights of $2 \mathrm{~mm}$ and $4 \mathrm{~mm}$ above the PCB surface respectively with the sample grid spacing $2 \mathrm{~mm}$. Each plane is measured with $26 \times 41$ sampling points. The induced voltage of the probe is recorded at $4 \mathrm{GSa} /$ second using a multi-channel digital oscilloscope. Adopting the PF, the voltage output of the probe can be converted to the magnetic field.

The tangential magnetic fields $H_{x}(t)$ and $H_{y}(t)$ in $x y$ plane at two scanning heights are shown in Fig. 16 to Fig. 19. The full-wave simulated, measured, and estimated tangential magnetic fields from the PCB are compared. Three sets of equivalent dipole model with $5 \times 9,8 \times 16$, and $11 \times 21$ dipole array respectively over the ground plane are used to replace the sources as shown in Fig. 15. For example, in Fig. 15(b), in the $x$-direction, the 5 dipoles are equidistantly placed from $0.01 \mathrm{~m}$ to $0.04 \mathrm{~m}$. In the $y$-direction, the 9 dipoles are equidistantly placed from $0.01 \mathrm{~m}$ to $0.07 \mathrm{~m}$. The dipole moments are all estimated using the scanned fields at a height of $2 \mathrm{~mm}$.
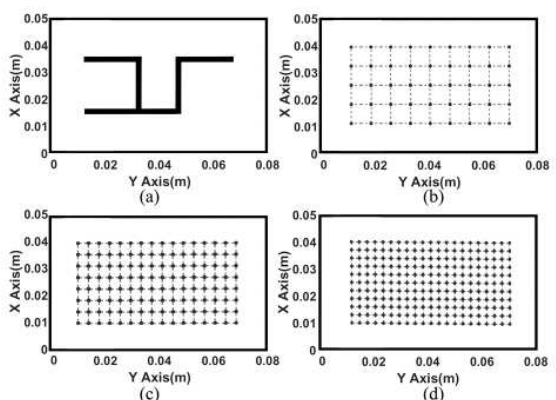

Fig. 15. Equivalent dipole model. (a) The PCB with several microstrips. (b) 5 $\times 9$ dipole array. (c) $8 \times 16$ dipole array. (d) $11 \times 21$ dipole array.

In Fig. 16, the measured tangential magnetic fields where the equivalent dipole model derived from are shown in the subfigures (c) and (d). The full-wave simulated tangential magnetic fields are also given as references in the subfigures (a) and (b) to validate the measured and estimated results. For illustration, two different sampling time points, $\mathrm{t} 1=21.3 \mathrm{~ns}$ and $\mathrm{t} 2=25.6 \mathrm{~ns}$, are selected to present the results.
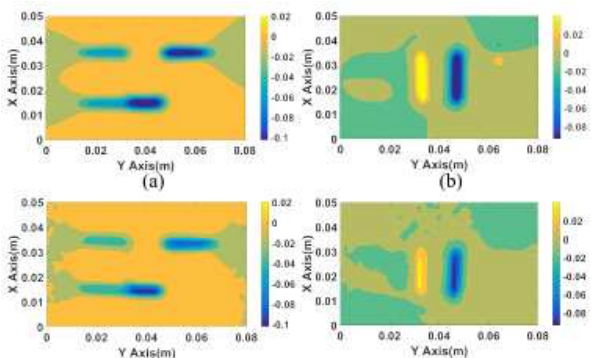

(d)
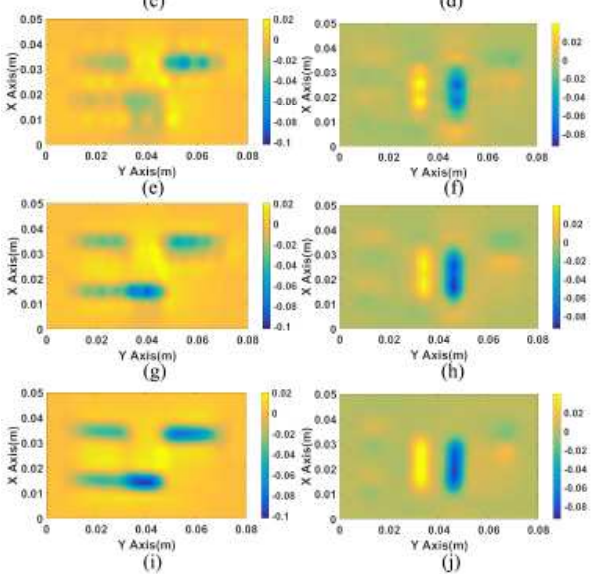

Fig. 16. Tangential magnetic fields $(\mathrm{A} / \mathrm{m})$ in $x y$ plane at $\mathrm{t}=21.3 \mathrm{~ns}$ at scanning height $2 \mathrm{~mm}$ above the PCB surface. (a) Full-wave simulated $H_{x}(t)$. (b) Full-wave simulated $H_{y}(t)$. (c) Measured $H_{x}(t)$. (d) Measured $H_{y}(t)$. (e) Estimated $H_{x}(t)$ calculated from $5 \times 9$ dipoles array. (f) Estimated $H_{y}(t)$ calculated from $5 \times 9$ dipoles array. (g) Estimated $H_{x}(t)$ calculated from $8 \times 16$ dipoles array. (h) Estimated $H_{y}(t)$ calculated from $8 \times 16$ dipoles array. (i) Estimated $H_{x}(t)$ calculated from $11 \times 21$ dipoles array. (j) Estimated $H_{y}(t)$ calculated from $11 \times 21$ dipoles array.

The reconstruction results from different dipole arrays indicate that the number of dipoles can affect the intensities and the distribution of the predicted magnetic fields. The relative-errors obtained by (11) between the measured magnetic fields and the reconstructed ones are shown in Table II.

TABLE II

THE Relative-ERRors BetweEn THE MEASUREd MAGNetic FieldS AND THE RELATIVE-ERRORS BET WEEN THE MEASURED MAGNETIC FIELDS AND \begin{tabular}{rcr} 
SCANNING HEIGHT 2 MM ABOVE THE PCB SURFACE \\
\hline \hline & \multicolumn{1}{c}{$H_{x}(t)$} & $H_{y}(t)$ \\
\hline $5 \times 9$ dipole array & $23.44 \%$ & $21.79 \%$ \\
$8 \times 16$ dipole array & $14.70 \%$ & $15.59 \%$ \\
$11 \times 21$ dipole array & $7.38 \%$ & $8.10 \%$ \\
\hline \hline
\end{tabular}

From the relative-errors given in Table II, for this PCB, the $11 \times 21$ dipoles array can be a good replication of the sources to 
reproduce the magnetic fields. Therefore, the rest of the examples given in Fig. 17 to Fig. 19 are studied with the $11 \times$ 21 dipoles array.
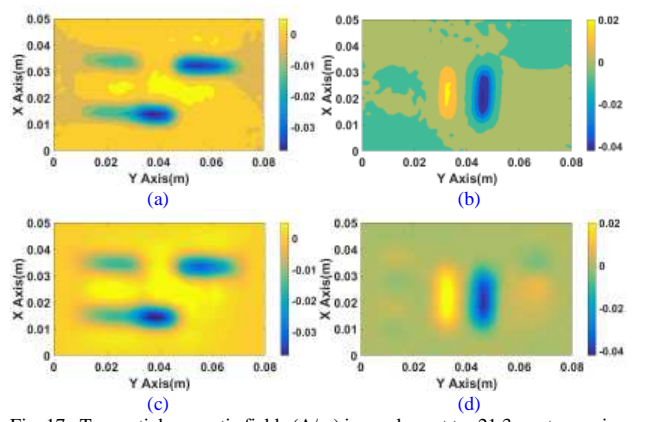

ds $(\mathrm{A} / \mathrm{m})$ in $x y$ plane at $\mathrm{t}=21.3 \mathrm{~ns}$ at scannin height $4 \mathrm{~mm}$ above the PCB surface. (a) Measured $H_{x}(t)$. (b) Measured $H_{y}(t)$ (c) Estimated $H_{x}(t)$. (d) Estimated $H_{y}(t)$. The fields in (c) and (d) are deduced from the dipole moments given by the fields at $2 \mathrm{~mm}$.
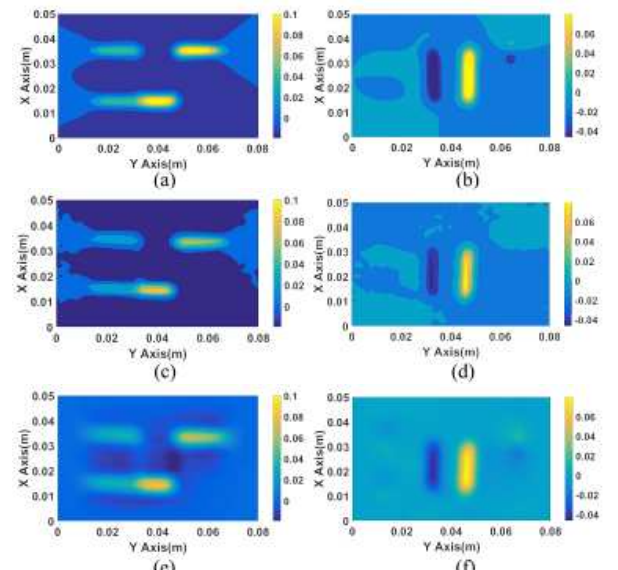

Fig. 18. Tangential magnetic fields $(\mathrm{A} / \mathrm{m})$ in $x y$ plane at $\mathrm{t}=25.6 \mathrm{~ns}$ at scanning height $2 \mathrm{~mm}$ above the PCB surface. (a) Full-wave simulated $H_{x}(t)$. (b) Full-wave simulated $H_{y}(t)$. (c) Measured $H_{x}(t)$. (d) Measured $H_{y}(t)$. (e) Estimated $H_{x}(t)$. (f) Estimated $H_{y}(t)$
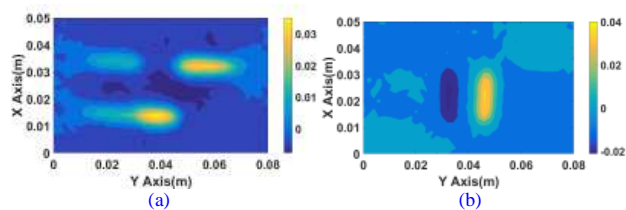

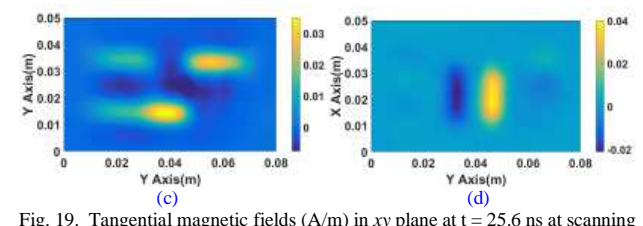

height $4 \mathrm{~mm}$ above PCB surface, (a) Measured $H_{x}(t)$. (b) Measured $H_{y}(t)$. (c) Estimated $H_{x}(t)$. (d) Estimated $H_{y}(t)$. The fields in (c) and (d) are deduced from the dipole moments given by the fields at $2 \mathrm{~mm}$.

Table III gives the relative-errors between the reconstructed fields and measured fields as given by (11)

TABLE III

THe Relative-Errors BetweEn THE MEASUREd Magnetic FieldS AND THE RECONSTRUCTED ONES FROM THE PCB WITH SEVERAL MICROSTRIPS

\begin{tabular}{llll}
\hline \hline & & $H_{x}(t)$ & $H_{y}(t)$ \\
\hline \multirow{2}{*}{ Scanning Height 2 mm } & $\mathrm{t}=21.3 \mathrm{~ns}$ & $7.38 \%$ & $8.10 \%$ \\
& $\mathrm{t}=25.6 \mathrm{~ns}$ & $8.97 \%$ & $7.55 \%$ \\
Scanning Height 4 mm & $\mathrm{t}=21.3 \mathrm{~ns}$ & $7.90 \%$ & $7.27 \%$ \\
& $\mathrm{t}=25.6 \mathrm{~ns}$ & $7.73 \%$ & $6.91 \%$ \\
\hline \hline
\end{tabular}

As the relative-errors shown in Table III, the equivalent dipole model derived from the lower scanning height not only can reconstruct the magnetic fields on the same plane, but also can well predict the tangential magnetic fields at higher scanning height.

From the results obtained in this section, the spatial-time distribution properties of the magnetic fields from the TL above a ground plane and the PCB with several microstrips can be intuitively observed. The accuracy of the near-field scanning procedure and the effectiveness of the equivalent dipole model are validated through the comparison among simulated, measured, and estimated magnetic fields.

In addition, from the view of computational efforts, the time-domain method is more computationally intensive than the frequency domain approach but compared with full-wave time-domain simulations, which can take several hours, the time-domain field estimations using dipole moments takes on the order of 77 seconds on a computer with processor Intel (R) Core (TM) i5-4460 CPU 3.2GHz.

\section{CONCLUSION}

In this paper, a near-field scanning system is established to measure the magnetic fields from a single TL above a ground plane and a PCB with several microstrips fully in the time-domain using a high-speed digital oscilloscope. The process for calibrating the magnetic field probes to obtain complex PFs is considered which is necessary during the experimental procedure. An innovative iterative scheme to estimate the time-domain sources that allows for retarded time in the time-domain has been validated. The measured tangential magnetic fields are used to derive equivalent source dipole models on the component surface of the DUTs to predict the emissions in other observation planes above the measurement plane. The ground planes of these two DUTs are also included in the model based on certain approximations. The equivalent 
dipoles are identified by fitting to the measured tangential magnetic fields. The agreement between measured and estimated results validates the effectiveness of the equivalent dipole model to predict the magnetic fields from DUTs.

Future work will include exciting the PCB with different time-domain sources simultaneously to show the reaction of the method to an unintentional radiator. It is also interesting to further investigate the used of the near-field scanning system to measure the fields from complex digital circuit board and DUT placed in semi-closed environment in time-domain and extend the equivalent dipole model to identify their equivalent sources.

\section{REFERENCES}

[1] A. D. Yaghjian, "An overview of near-field antenna measurements," IEEE Trans. Antennas Propagat., vol. 34, no. 1, pp. 30-45, Jan. 1986.

E. X. Liu, W. J. Zhao, B. F. Wang, and X. C. Wei, "Near-field scanning and its EMC applications," in Proc. Int. Symp. IEEE Electromagn. Compat., Washington DC, USA, Aug. 2017, pp. 327-332.

[3] X. Tong, D. W. P. Thomas, A. Nothofer, P. Sewell, and C. Christopoulos, "Modeling electrometic emissions from Christopoulos, "Moded Trans. Electromagn. Compat., vol. 52, no. 2, pp. 462-470, May 2010.

[4] X. Tong. D W. P. Thomes, A. Nothofer, P. Sewell, and C. C. Tong, D. W. P. Thomas, A. Nothofer, P. Sewell, and C. Christopoulos, "Reduction of sensitivity to measurement errors in the in Proc. IET 8th Int. Conf. Comput. Electromagn., Apr. 2011, pp. 1-2.

5] Q. Huang and J. Fan, "Machine learning based source reconstruction fo RF desense," IEEE Trans. Electromagn. Compat., vol. 60, no. 6, pp. 1640-1647, Dec. 2018

[6] W. Zhao, E. Liu, B. Wang, S. Gao, and C. Png, "Differential Evolutionary Optimization of an Equivalent Dipole Model for Electromagnetic Emission Analysis." IEEE Trans. Electromagn. Compat., vol. 60, no. 6, pp. 1635-1639, Dec. 2018.

[7] B. Wang, E. X. Liu, W. J. Zhao, and C. E. Png, "Reconstruction of equivalent emission sources for PCBs from near field scanning using a differential evolution algorithm," IEEE Trans. Electromagn. Compat. vol. 60, no. 6, pp. 1670-1677, Dec. 2018.

[8] J. Zhang and J. Fan, "Source reconstruction for IC radiated emissions based on magnitude-only near-field scanning," IEEE Trans. Electromagn. Compat., vol. 59, no. 2, pp. 557-566, Apr. 2017.

[9] Q. Huang, L. Li, X. Yan, B. Bae, H. Park, C. Hwang, and J. Fan, "MoM-based ground current reconstruction in RFI application," IEEE Trans. Electromagn. Compat., vol. 60, no. 4, pp. 1121-1128, Aug. 2018

[10] G. Gradoni, J. Russer, M. H. Baharuddin, M. Haider, P. Russer, C. Smartt, S. C. Creagh, G. Tanner and D. W. P. Thomas, "Stochastic electromagnetic field propagation - measurement and modelling" Phil. Trans. Roy. Soc. A, vol. 376, no. 2134, Oct, 2018

[11] S. A. Bokhari, J. R. Mosig, and F. E. Gardiol, "Radiation pattern computation of microstrip antennas on finite size ground planes", in IEE Proc. H, vol.139, no. 3, pp. 278-286, Jun. 1992.

[12] S. Pan, J. Kim, S. Kim, J. Park, H. Oh, and J. Fan, "An equivalent three dipole model for IC radiated emissions based on TEM cell measurement," in Proc. IEEE Int. Symp. Electromagn. Compat., For Lauderdale, FL, USA, Jul. 25-30, 2010, pp. 652-656.

[13] M. J. Basford, C. Smartt, D. W. P. Thomas, and S. Greedy, "On the disruption of wired serial communication links by time domai interference," in Proc. IEEE Int. Symp. Electromagn. Compat. Asia-Pacific Symp. Electromagn. Compat. (EMC/APEMC), May 2018, pp. $183-186$

[14] G. Costa, M. Pous, A. Atienza, and F. Silva, "Time-domai electromagnetic interference measurement system for intermitten disturbances," in Proc. Int. Symp. Electromagn. Compat., 2014, pp. 833-837.

[15] B. Zitouna, and J. B. H. Slama, "Enhancement of Time-Domain Electromagnetic Inverse Method for Modeling Circuits Radiations," IEEE Trans. Electromagn. Compat., vol. 58, no. 2, pp. 534-542, Ap 2016

[16] L. Zhang, Y. Du, Z. Liu, and A. Cao, "Prediction of PCB electromagnetic emission based on improved equivalent electric dipoles in time domain,"
[17] D. W. P. Thomas, C. Smartt, and S. Greedy, "Characterisation of radiated fields from PCBs in the time domain," in Proc. 2015 Int Symp. Electromagn. Compat., Dresden, Germany, 2015, pp. 947-952.

[18] Y. Liu, B. Ravelo, and A. K. Jastrzebski, "Time-domain magnetic dipole model of PCB near-field emission," IEEE Trans. Electromagn. Compat. vol. 58 , no. 5, pp. 1561-1569, Oct. 2016.

19] B. Vrignon, K. Abouda, A. Doridant, and N. Baptistat, "Time-domain measurements using near field scanning method for fast transient current reconstruction," in Proc. Int. Workshop Electromagn. Compat. Integr. Circuits (EMCCompo), St. Petersburg, Russia, 2017, pp. 119-124.

20] M. Serhir, "Transient UWB antenna near-field and far-field assessment from time domain planar near-field characterization: Simulation and measurement investigations," IEEE Trans. Antennas Propag., vol. 63. no. 11 , pp. $4868-4876$, Nov. 2015

[21] M. H. Francis, "IEEE recommended practice for near field antenna measurements," IEEE Std 1720-2012, New York, NY, USA, IEEE, 2012.

22] LANGER-EMV Technik, "RF-R50-1", [Online]. Available: https://www.langer-emv.de/en/product/rf-r-50-1-h-field-probe-30-mhzup-to-3-ghz

[23] LANGER-EMV Technik, "RF-R0.3-3", [Online]. Available: https://www.langer-emv.de/en/product/rf-r-0-3-3-h-field-probe-mini-30 -mhz-up-to-3-ghz 